\title{
La vigilancia epidemiológica desde la NOM-017-SSA2-2012
}

\section{Epidemiological surveillance from NOM-017-SSA2-2012}

\author{
Annette M. Ramirez-Guzman ${ }^{a}$, Yadira Hernández-Reyes ${ }^{b}$, Estefanía Meneces-Mera ${ }^{c}$, \\ Diana V. Sanchez-Martinez ${ }^{d}$
}

\begin{abstract}
:
In Mexico, through the National Epidemiological Surveillance System (SINAVE) a systematic, continuous, timely and reliable collection is carried out, which contains essential information to know the health conditions of the population and its determinants. The analysis and interpretation of this information allows decision-making. The actions necessary for epidemiological surveillance and laboratory diagnosis are described in manuals with some validity, since the organization and operation of SINAVE, as well as training and research is described in NOM-017-SSA2-2012, which are the basis for establishing public health policies and programs.
\end{abstract}

This standard establishes the criteria, specifications and operating guidelines of SINAVE and is mandatory throughout the national territory, therefore it involves the public, social and private sectors that make up the National Health System.

\section{Keywords:}

SINAVE, NOM-017-SSA2-2012, Public Health, National system of health.

\section{Resumen:}

En México, a través del Sistema Nacional de Vigilancia Epidemiológica (SINAVE) se lleva a cabo una recolección sistemática, continua, oportuna y confiable, que contiene información esencial para conocer las condiciones de salud de la población y sus determinantes. El análisis e interpretación de esa información permite la toma de decisiones. Las acciones necesarias para la vigilancia epidemiológica y el diagnóstico de laboratorio se describen en manuales con cierta vigencia, pues la organización y funcionamiento de SINAVE, así como la capacitación e investigación se describen en la NOM-017-SSA2-2012, las cuales son la base para establecer políticas y programas de salud pública.

Esta norma establece los criterios, especificaciones y directrices de operación del SINAVE y es obligatoria en todo el territorio nacional, por lo tanto involucra al sector público, social y privado que integran el Sistema Nacional de Salud.

\section{Palabras Clave:}

SINAVE, NOM-017-SSA2-2012, Salud Pública, Sistema Nacional de Salud.

\section{Síntesis}

La Norma Oficial Mexicana NOM-017-SSA2-2012, para la vigilancia epidemiológica es una regulación técnica de observancia obligatoria, con la finalidad de establecer criterios especificaciones y directrices de operación del Sistema Nacional de Vigilancia Epidemiológica, para la recolección sistemática, continua, oportuna y confiable de información relevante y necesaria sobre las condiciones de salud de la población y sus determinantes (DOF, 2013).

Esta norma habla específicamente sobre la coordinación de diferentes organismos para vigilar el buen desarrollo de los procesos epidemiológicos.

\footnotetext{
${ }^{a}$ Universidad Autónoma del Estado de Hidalgo, https://orcid.org/0000-0002-4258-3297, Email: ra450391@uaeh.edu.mx

b Universidad Autónoma del Estado de Hidalgo, https://orcid.org/0000-0001-8110-2823, Email: he440195@uaeh.edu.mx

c Universidad Autónoma del Estado de Hidalgo, https://orcid.org/0000-0003-1408-0964, Email: me449481@uaeh.edu.mx

d Autor de Correspondencia, Universidad Autónoma del Estado de Hidalgo, https://orcid.org/0000-0002-7660-7234, Email:
} 
La vigilancia epidemiológica es un sistema que realiza la recolección sobre los diversos eventos de intereses medico epidemiológico capaz de analizar la información y proporcionar un panorama solido que permita iniciar, profundizar o rectificar acciones de prevención y control.

Durante la vigilancia epidemiológica se realiza la recolección veraz, oportuna, verídica de información indispensable sobre las condiciones de salud de la población y sus determinantes. La Secretaría de Salud es el órgano rector del SINAVE (Sistema Nacional de Vigilancia Epidemiológica.) y actúa como la instancia responsable de recopilar, procesar y dar a conocer toda la información generada por el SNS (Sistema Nacional de Salud).

La coordinación de los mecanismos de vigilancia diagnóstico y referencia epidemiológicos, se ejerce por conducto de la Dirección General de Epidemiologia (DGE) en coordinación con los diferentes sectores de SNS. (SSa, 2020)

Las acciones de vigilancia epidemiológica se realizan a nivel federal, estatal, jurisdiccional, local y sus equivalentes en distintas instituciones que conforman el SNS.

Los comités de vigilancia epidemiológica son:

- COJUVE: Comité Jurisdiccional de Vigilancia Epidemiológica. (jurisdiccional).

- CEVE: Comité Estatal de Vigilancia Epidemiológica, es la instancia responsable de difundir y verificar el cumplimiento de los procedimientos de la vigilancia epidemiológica en el estado, además es el órgano coordinador del SINAVE en las entidades federativas.

- CONAVE: Comité Nacional para la Vigilancia Epidemiológica, es la instancia responsable de unificar y homologar los criterios procedimientos y contenidos para el funcionamiento de la vigilancia epidemiológica del país, conforme a la normatividad vigente.

Los componentes de la vigilancia epidemiológica
-Vigilancia de la mortalidad

-Vigilancia de la morbilidad

-Vigilancia especial

- Vigilancia de emergencias en salud publica

-Vigilancia internacional

En la norma se mencionan las metodologías y procedimientos para la vigilancia epidemiológica (DOF, 2013).

-Vigilancia convencional.

- Estudio de caso.

-Estudio de brote.

- Registros nominales.

- Búsqueda activa de casos.

- Red negativa.

-Vigilancia basada en laboratorio.

-Vigilancia centinela

-Vigilancia sindromática.

-Encuestas.

-Vigilancia activa de la mortalidad.

-Autopsias verbales.

-Vigilancia comunitaria.

- Vigilancia nosocomial.

-Dictaminación por grupo de expertos.

-Evaluación y análisis de riesgos y determinantes de la salud.

La notificación, llenado del estudio epidemiológico y aseguramiento de la toma de muestra es responsabilidad del médico tratante, la prioridad de la notificación para la vigilancia epidemiológica debe ser inmediata diaria o seminal (SSa, 2020). La autoridad sanitaria debe llevar a cabo la evaluación y análisis de riesgo y determinantes de la salud.

La información obtenida a través de la vigilancia epidemiológica debe difundirse a través de reportes impresos o electrónicos que favorezcan la accesibilidad a los datos. 


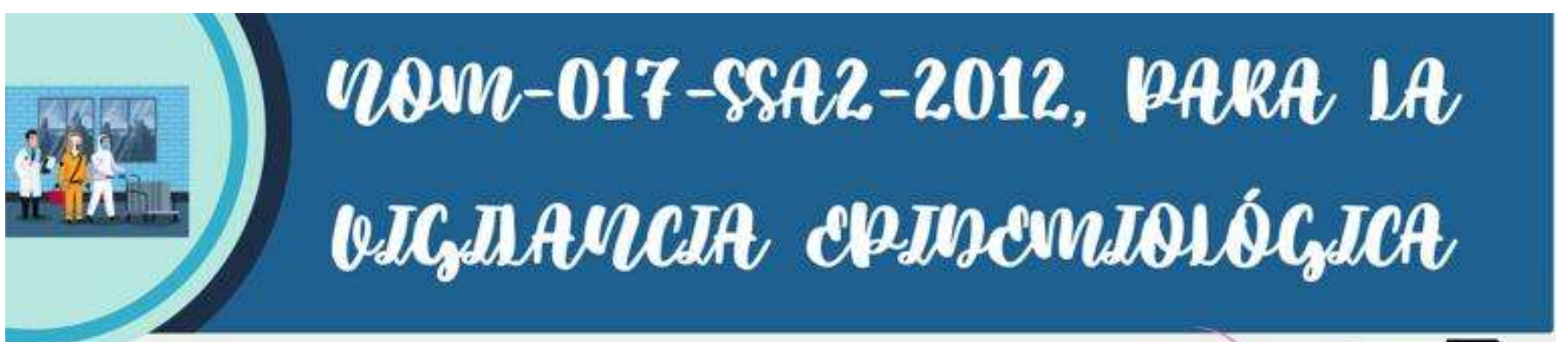

ESTABBLECEE $\rightarrow$ Criterios, especificaciones y directrices de operación.

SINAVE ....... Información …… Sistema Nacional de Salud
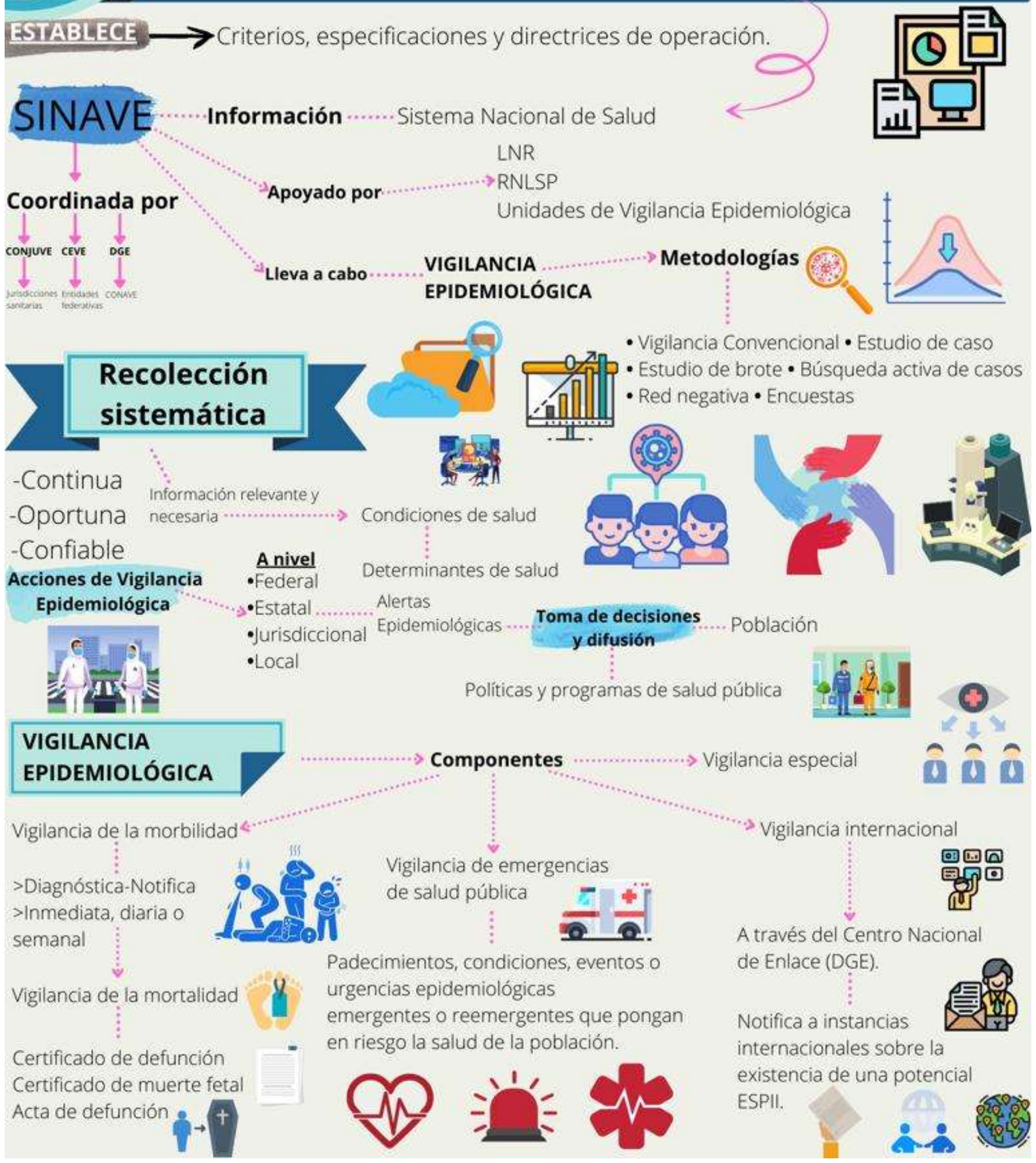


\section{Referencias}

[1] DOF. (19 de febrero de 2013). Obtenido de NORMA Oficial Mexicana NOM-017-SSA2-2012, Para la vigilancia epidemiológica.:

http://dof.gob.mx/nota_detalle.php?codigo $=5288225 \&$ fecha $=19 / 02 / 2013 \#: \sim:$ text=Esta\%20Norma\%20Oficial\%20Mexic ana\%20establece,la\%20poblaci\%C3\%B3n\%20y\%20sus\%2 Odeterminantes.

[2] Secretaría de Salud. (15 de enero de 2020). Obtenido de Sistema Nacional de Vigilancia Epidemiológica: https://www.gob.mx/salud/acciones-y-

programas/sistema-nacional-de-vigilanciaepidemiologica 